\title{
Liouville-type theorem for some nonlinear systems in a half-space
}

\author{
Linfen Cao ${ }^{1 *}$, Zhaohui Dai ${ }^{2}$ and Wenyan $\mathrm{Li}^{3}$
}

\section{"Correspondence:}

caolf2010@yahoo.com

'College of Mathematics and Information Science, Henan Normal

University, Xinxiang, Henan 453007, P.R. China

Full list of author information is available at the end of the article

\begin{abstract}
In this paper we consider the following Hardy-Littlewood-Sobolev (HLS)-type system of nonlinear equations in the half-space $R_{+}^{n}: u(x)=\int_{R_{+}^{n}}\left(\frac{1}{|x-y|^{n-\alpha}}-\frac{1}{\left|x^{*}-y\right|^{n-\alpha}}\right) v^{q}(y) d y$, $v(x)=\int_{R_{+}^{n}}\left(\frac{1}{|x-y|^{n-\alpha}}-\frac{1}{\left|x^{*}-y\right|^{n-\alpha}}\right) u^{p}(y) d y$, where $p, q>1$ and $x^{*}$ is the reflection of $x$ about the boundary $\left\{x_{n}=0\right\}$. By using the method of moving planes in integral forms, we obtain monotonicity of the positive solution of the integral equations system of the abstract in three cases: the so-called subcritical, critical, and supercritical cases, and we obtain a new Liouville-type theorem of this system under some integrability conditions. In particular, our results unify and generalize many cases of Liouville-type theorems in (Cao and Dai in J. Math. Anal. Appl. 389:1365-1373, 2012; Cao and Dai in J. Inequal. Appl. 2013:37, 2013) and (Li et al. in Complex Var. Elliptic Equ. 2013, doi:10.1080/17476933.2013.854346).
\end{abstract}

MSC: 35B05; 35B45

Keywords: Liouville-type theorem; HLS inequality; systems of integral equations; monotonicity; moving planes method in integral forms

\section{Introduction}

In [1], Chen and Li discussed the HLS-type system of nonlinear equations in the whole space $R^{n}$ :

$$
\left\{\begin{array}{l}
u(x)=\int_{R^{n}} \frac{1}{|x-y|^{n-\alpha}} v^{q}(y) d y, \\
v(x)=\int_{R^{n}} \frac{1}{|x-y|^{n-\alpha}} u^{p}(y) d y, \quad x \in R^{n} .
\end{array}\right.
$$

By the method of moving planes in integral forms they derived that the positive solutions of (1.1) are radially symmetric and such solutions are nonexistent under some integrability conditions.

In a recent paper of Chen and Li [2], the equivalence between integral equation (1.1) and the following PDEs was established:

$$
\left\{\begin{array}{l}
(-\triangle)^{\frac{\alpha}{2}} u(x)=v^{q}(x), \\
(-\triangle)^{\frac{\alpha}{2}} v(x)=u^{p}(x), \quad x \in R^{n},
\end{array}\right.
$$

where $\alpha$ is any even number between 0 and $n$. In fact, their equivalence results are more general than above. Such an equivalence provides a technique for studying the PDEs: one can use the corresponding integral equations to investigate the global properties.

@2014 Cao et al.; licensee Springer. This is an Open Access article distributed under the terms of the Creative Commons Attribution License (http://creativecommons.org/licenses/by/2.0), which permits unrestricted use, distribution, and reproduction in any medium, provided the original work is properly cited. 
In this paper we want to generalize monotonicity and nonexistence results of positive solutions of an HLS-type system in the whole space $R^{n}$ to ones in a half-space.

Let $R_{+}^{n}$ be the upper half Euclidean space

$$
R_{+}^{n}=\left\{x=\left(x_{1}, \ldots, x_{n}\right) \in R^{n} \mid x_{n}>0\right\} .
$$

For convenience we introduce the function $G(x, y, \alpha)$ in this paper

$$
G(x, y, \alpha)=\frac{1}{|x-y|^{n-\alpha}}-\frac{1}{\left|x^{*}-y\right|^{n-\alpha}},
$$

then the integral system of the abstract can be rewritten as follows:

$$
\left\{\begin{array}{l}
u(x)=\int_{R_{+}^{n}} G(x, y, \alpha) v^{q}(y) d y, \\
v(x)=\int_{R_{+}^{n}} G(x, y, \alpha) u^{p}(y) d y, \quad \forall x \in R_{+}^{n} .
\end{array}\right.
$$

The integral system (1.4) is usually divided into three cases according to the value of the exponents $(p, q)$. We say that system (1.4) is in the critical case when the pair $(p, q)$ satisfies the relation

$$
\frac{1}{q+1}+\frac{1}{p+1}=\frac{n-\alpha}{n}
$$

it is in the supercritical case when '<' holds; and in the subcritical case when '>' holds, i.e.

$$
\frac{1}{q+1}+\frac{1}{p+1}>\frac{n-\alpha}{n}
$$

In [3], the first and the second authors concluded to the nonexistence of (1.4) in the critical case.

Theorem 1.1 ([3]) Let $(u, v)$ be a pair of positive solutions of (1.4) in the critical case (1.5). Assume that $u \in L^{p+1}\left(R_{+}^{n}\right)$ and $v \in L^{q+1}\left(R_{+}^{n}\right)$, then both $u$ and $v$ are strictly monotonically increasing with the variable $x_{n}$.

Theorem $1.2([3])$ Let $(u, v)$ be a pair of positive solutions of (1.4) with the critical case (1.5). Assume that $u \in L^{p+1}\left(R_{+}^{n}\right)$ and $v \in L^{q+1}\left(R_{+}^{n}\right)$ are nonnegative, then $u=v \equiv 0$.

In this paper, we further consider the nonnegative solution of the integral equations system (1.4) by using the method of moving planes in integral forms. We prove that the positive solution pair $(u, v)$ of $(1.4)$ is strictly monotonically increasing with respect to the variable $x_{n}$.

Theorem 1.3 Assume that $1<p, q<\infty$, and that there exist $p_{1} \geq 1$ and $q_{1} \geq 1$ such that

$$
\begin{aligned}
& \frac{p-1}{p_{1}}+\frac{q-1}{q_{1}} \geq \frac{2 \alpha}{n}, \\
& \frac{p-1}{p_{1}}, \frac{q-1}{q_{1}}<1,
\end{aligned}
$$




$$
\frac{p}{p_{1}}, \frac{q}{q_{1}}>\frac{\alpha}{n}
$$

Suppose that $u \in L^{p_{1}}\left(R_{+}^{n}\right)$ and $v \in L^{q_{1}}\left(R_{+}^{n}\right)$ is a pair of positive solutions of integral system (1.4), then both $u$ and $v$ are strictly monotonically increasing with respect to the variable $x_{n}$.

Theorem 1.3 yields the main result of the paper.

Theorem 1.4 Let $(u, v)$ be a pair of positive solutions of $(1.4)$ with $1<p, q<\infty$, and let there exist $p_{1} \geq 1$ and $q_{1} \geq 1$ such that (1.7), (1.8), and (1.9) hold. Assume that $u \in L^{p_{1}}\left(R_{+}^{n}\right)$ and $v \in L^{q_{1}}\left(R_{+}^{n}\right)$ are nonnegative, then $u=v \equiv 0$.

To prove Theorem 1.4, we will use the method of moving planes in integral forms to obtain the monotonicity of the positive solutions of system (1.4). Corresponding to the half-space problem (1.4), the Liouville-type Theorem 1.4 for the whole space problem (1.1) was established by Chen and $\mathrm{Li}[1]$.

Remark 1 Theorem 1.4 concerning monotonicity and nonexistence of solutions is true in all three cases: subcritical, critical, and supercritical.

Remark 2 Theorem 1.4 unifies and generalizes some Liouville-type results of positive solutions of other integral systems. In particular, we find some examples to show the existence of such pairs of $\left(p_{1}, q_{1}\right)$ that satisfy all these conditions (1.7), (1.8), and (1.9) in Theorem 1.4 .

\section{Preliminaries}

In this section, we introduce some lemmas as preliminaries.

For $x, y \in R_{+}^{n}$, define

$$
G(x, y, \alpha)=\frac{1}{|x-y|^{n-\alpha}}-\frac{1}{\left|x^{*}-y\right|^{n-\alpha}},
$$

where $x^{*}=\left(x_{1}, \ldots, x_{n-1},-x_{n}\right)$ is the reflection of the point $x$ about the $\partial R_{+}^{n}$.

Let $\lambda$ be a positive real number. Define

$$
\begin{aligned}
& \Sigma_{\lambda}=\left\{x=\left(x_{1}, x_{2}, \ldots, x_{n}\right) \in R^{n} \mid 0<x_{n}<\lambda\right\}, \\
& T_{\lambda}=\left\{x \in R_{+}^{n} \mid x_{n}=\lambda\right\}, \quad \Sigma_{\lambda}^{C}=R_{+}^{n} \backslash \Sigma_{\lambda} .
\end{aligned}
$$

Let

$$
x^{\lambda}=\left(x_{1}, x_{2}, \ldots, x_{n-1}, 2 \lambda-x_{n}\right)
$$

be the reflection of the point $x=\left(x_{1}, x_{2}, \ldots, x_{n}\right)$ about the plane $T_{\lambda}$.

The following lemma states some properties of the function $G(x, y, \alpha)$.

Lemma 2.1 (Lemma 2.1 in [4])

(i) For any $x, y \in \Sigma_{\lambda}, x \neq y$, we have

$$
G\left(x^{\lambda}, y^{\lambda}, \alpha\right)>\max \left\{G\left(x^{\lambda}, y, \alpha\right), G\left(x, y^{\lambda}, \alpha\right)\right\}
$$


and

$$
G\left(x^{\lambda}, y^{\lambda}, \alpha\right)-G(x, y, \alpha)>\left|G\left(x^{\lambda}, y, \alpha\right)-G\left(x, y^{\lambda}, \alpha\right)\right|
$$

(ii) For any $x \in \Sigma_{\lambda}, y \in \Sigma_{\lambda}^{C}$, we have

$$
G\left(x^{\lambda}, y, \alpha\right)>G(x, y, \alpha)
$$

Lemma 2.2 (Lemma 3.1 in [3]) Let $(u, v)$ be any pair of positive solutions of (1.4), for any $x \in \Sigma_{\lambda}$, we have

$$
\begin{aligned}
& u(x)-u_{\lambda}(x) \leq \int_{\Sigma_{\lambda}}\left[G\left(x^{\lambda}, y^{\lambda}, \alpha\right)-G\left(x, y^{\lambda}, \alpha\right)\right]\left[v^{q}(y)-v_{\lambda}^{q}(y)\right] d y, \\
& v(x)-v_{\lambda}(x) \leq \int_{\Sigma_{\lambda}}\left[G\left(x^{\lambda}, y^{\lambda}, \alpha\right)-G\left(x, y^{\lambda}, \alpha\right)\right]\left[u^{p}(y)-u_{\lambda}^{p}(y)\right] d y .
\end{aligned}
$$

In addition, we need the equivalent form of the Hardy-Littlewood-Sobolev inequality.

Lemma 2.3 (Classical HLS inequality) Let $g \in L^{\frac{n r}{n+\alpha p}}\left(R^{n}\right)$ for $\frac{n}{n-\alpha}<r<\infty$. Define

$$
\operatorname{Tg}(x)=\int_{R^{n}} \frac{1}{|x-y|^{n-\alpha}} g(y) d y .
$$

Then

$$
\|\operatorname{Tg}\|_{L^{r}\left(R^{n}\right)} \leq C(n, \alpha, r)\|g\|_{L^{\frac{n r}{n+\alpha r}\left(R^{n}\right)}} .
$$

\section{Proof of main theorems}

In this section, by the method of moving planes in integral forms we derive the nonexistence of positive solutions to the integral system (1.4) and obtain a new Liouville-type theorem in a half-space. To prove the theorem, we need some proper match of the exponents in the involving integrals, which will be prepared in Part 1 . The moving of planes will be carried out in Part 2.

\section{Part 1. The preparations.}

Step 1. For convenience, we may assume equality in (1.7):

$$
\frac{p-1}{p_{1}}-\frac{\alpha}{n}+\frac{q-1}{q_{1}}-\frac{\alpha}{n}=0
$$

by increasing $p_{1}$ and $q_{1}$ to $\bar{p}_{1}$ and $\bar{q}_{1}$ while still (1.8) and (1.9) hold. To see this, let $\left(p_{1}, q_{1}\right)$ be the pair where the three inequalities (1.7), (1.8), and (1.9) holds. Obviously, (1.8) remains true by increasing $p_{1}$ and $q_{1}$. If we continuously increase $p_{1}$ up to $p_{2}$ and $q_{1}$ up to $q_{2}$ until the strict inequality (1.9) becomes the equality:

$$
\frac{p}{p_{2}}=\frac{\alpha}{n}=\frac{q}{q_{2}}
$$

then we would have

$$
\frac{p-1}{p_{1}}-\frac{\alpha}{n}+\frac{q-1}{q_{1}}-\frac{\alpha}{n}=-\frac{1}{p_{2}}-\frac{1}{q_{2}}<0 .
$$


It follows from the intermediate value theorem that there exist $\bar{p}_{1} \in\left(p_{1}, p_{2}\right)$ and $\bar{q}_{1} \in$ $\left(q_{1}, q_{2}\right)$, such that the equality (3.1) holds with $p_{1}$ and $q_{1}$ replaced by $\bar{p}_{1}$ and $\bar{q}_{1}$ while (1.9) remains true. Hence, without loss of generality and for simplicity, in the next step, we may assume (3.1).

Step 2. Under the conditions of the theorem, there exist two non-empty open intervals $I_{s}$ and $I_{r}$ such that for any $r \in I_{r}$ and a corresponding $s \in I_{s}$, we have

$$
\begin{aligned}
& r \geq p_{1} \quad \text { and } \quad s \geq q_{1}, \\
& r, s>\frac{n}{n-\alpha}, \\
& \frac{n+\alpha r}{n r}=\frac{q-1}{q_{1}}+\frac{1}{s} \text { and } \quad \frac{n+\alpha s}{n s}=\frac{p-1}{p_{1}}+\frac{1}{r} .
\end{aligned}
$$

We have

$$
\frac{1}{s}<\frac{\alpha}{n}+\frac{1}{r} \text { and } \frac{1}{r}<\frac{\alpha}{n}+\frac{1}{s} .
$$

Remark The proof of Part 1 is the same as the proof in [1].

\section{Part 2. The method of moving planes.}

To prove Theorem 1.3, we compare $(u(x), v(x))$ and $\left(u_{\lambda}(x), v_{\lambda}(x)\right)$ on $\Sigma_{\lambda}$. The proof consists of two steps.

In the first step, we start from the very lower end of our region $R_{+}^{n}$, i.e. near $x_{n}=0$. We will show that for $\lambda$ sufficiently small,

$$
u_{\lambda}(x) \geq u(x) \quad \text { and } \quad v_{\lambda}(x) \geq v(x), \quad \forall x \in \Sigma_{\lambda} .
$$

In the second step, we will move our plane $T_{\lambda}$ toward the positive direction of the $x_{n}$-axis as long as the inequality (3.6) holds.

Step 1 . Define

$$
\Sigma_{\lambda}^{u}=\left\{x \mid x \in \Sigma_{\lambda}, u(x)>u_{\lambda}(x)\right\}
$$

and

$$
\Sigma_{\lambda}^{v}=\left\{x \mid x \in \Sigma_{\lambda}, v(x)>v_{\lambda}(x)\right\} .
$$

We show that for sufficiently small positive $\lambda, \Sigma_{\lambda}^{u}$, and $\Sigma_{\lambda}^{v}$ must have measure zero. In fact, by Lemma 2.2 , it is easy to verify that

$$
\begin{aligned}
u(x)-u_{\lambda}(x) \leq & \int_{\Sigma_{\lambda}}\left[G\left(x^{\lambda}, y^{\lambda}, \alpha\right)-G\left(x, y^{\lambda}, \alpha\right)\right]\left(v^{q}(y)-v_{\lambda}^{q}(y)\right) d y \\
= & \int_{\Sigma_{\lambda} \backslash \Sigma_{\lambda}^{\nu}}\left[G\left(x^{\lambda}, y^{\lambda}, \alpha\right)-G\left(x, y^{\lambda}, \alpha\right)\right]\left(v^{q}(y)-v_{\lambda}^{q}(y)\right) d y \\
& +\int_{\Sigma_{\lambda}^{\nu}}\left[G\left(x^{\lambda}, y^{\lambda}, \alpha\right)-G\left(x, y^{\lambda}, \alpha\right)\right]\left(v^{q}(y)-v_{\lambda}^{q}(y)\right) d y \\
\leq & \int_{\Sigma_{\lambda}^{\nu}}\left[G\left(x^{\lambda}, y^{\lambda}, \alpha\right)-G\left(x, y^{\lambda}, \alpha\right)\right]\left(v^{q}(y)-v_{\lambda}^{q}(y)\right) d y
\end{aligned}
$$




$$
\begin{aligned}
& \leq \int_{\Sigma_{\lambda}^{\nu}} G\left(x^{\lambda}, y^{\lambda}, \alpha\right)\left[v^{q}(y)-v_{\lambda}^{q}(y)\right] d y \\
& \leq q \int_{\Sigma_{\lambda}^{v}} \frac{1}{|x-y|^{n-\alpha}} \psi_{\lambda}^{q-1}(y)\left[v(y)-v_{\lambda}(y)\right] d y \\
& \leq q \int_{\Sigma_{\lambda}^{v}} \frac{1}{|x-y|^{n-\alpha}} v^{q-1}(y)\left[v(y)-v_{\lambda}(y)\right] d y,
\end{aligned}
$$

where $\psi_{\lambda}(y)$ is valued between $v(y)$ and $v_{\lambda}(y)$; therefore on $\Sigma_{\lambda}^{\nu}$, we have

$$
0 \leq v_{\lambda}(y) \leq \psi_{\lambda}(y) \leq v(y)
$$

Let $(r, s)$ be a pair of numbers that satisfy (3.2)-(3.5). It follows from the Hardy-LittlewoodSobolev inequality (2.1) that

$$
\left\|u_{\lambda}-u\right\|_{L^{r}\left(\Sigma_{\lambda}^{u}\right)} \leq C\left\|v^{q-1}\left(v_{\lambda}-v\right)\right\|_{L^{(n r) /(n+\alpha r)}\left(\Sigma_{\lambda}^{\nu}\right)} .
$$

Then by the Hölder inequality,

$$
\left\|u_{\lambda}-u\right\|_{L^{r}\left(\Sigma_{\lambda}^{u}\right)} \leq C\|v\|_{L^{q_{1}\left(\Sigma_{\lambda}^{v}\right)}}^{q-1}\left\|\nu_{\lambda}-v\right\|_{L^{s}\left(\Sigma_{\lambda}^{\nu}\right)} .
$$

Similarly, one can show that

$$
\left\|\nu_{\lambda}-v\right\|_{L^{s}\left(\Sigma_{\lambda}^{\nu}\right)} \leq C\|u\|_{L^{p_{1}}\left(\Sigma_{\lambda}^{u}\right)}^{p-1}\left\|u_{\lambda}-u\right\|_{L^{r}\left(\Sigma_{\lambda}^{u}\right)} .
$$

Combining (3.7) and (3.8), we arrive at

$$
\left\|u_{\lambda}-u\right\|_{L^{r}\left(\Sigma_{\lambda}^{u}\right)} \leq C\|v\|_{L^{q_{1}}\left(\Sigma_{\lambda}^{v}\right.}^{q-1}\|u\|_{L^{p_{1}}\left(\Sigma_{\lambda}^{u}\right)}^{p-1}\left\|u_{\lambda}-u\right\|_{L^{r}\left(\Sigma_{\lambda}^{u}\right)} .
$$

By the conditions that $u \in L^{p_{1}}\left(R_{+}^{n}\right)$ and $v \in L^{q_{1}}\left(R_{+}^{n}\right)$, we can choose sufficiently small positive $\lambda$, such that

$$
C\|v\|_{L^{q_{1}\left(\Sigma_{\lambda}^{v}\right)}}^{q-1}\|u\|_{L^{p_{1}\left(\Sigma_{\lambda}^{u}\right)}}^{p-1} \leq \frac{1}{2} .
$$

Now inequality (3.9) implies $\left\|u_{\lambda}-u\right\|_{L^{p+1}\left(\Sigma_{\lambda}^{u}\right)}=0$, and therefore $\Sigma_{\lambda}^{u}$ must have measure zero. Similarly, one can show that $\Sigma_{\lambda}^{v}$ has measure zero. Therefore (3.6) holds. This completes Step 1.

Step 2. (Move the plane to the limiting position to derive symmetry and monotonicity.) Inequality (3.6) provides a starting point to move the plane $T_{\lambda}$. Now we start from the neighborhood of $x_{n}=0$ and move the plane up as long as (3.6) holds to the limiting position. We will show that the solution $u(x)$ and $v(x)$ must be symmetric about the limiting plane and be strictly monotonically increasing with respect to $x_{n}$. More precisely, define

$$
\lambda_{0}=\sup \left\{\lambda \mid u(x) \leq u_{\mu}(x) \text { and } v(x) \leq v_{\mu}(x), \forall x \in \Sigma_{\mu}, \mu \leq \lambda\right\} .
$$

Suppose that for such a $\lambda_{0}$, we will show that both $u(x)$ and $v(x)$ must be symmetric about the plane $T_{\lambda_{0}}$ by using a contradiction argument. Assume that on $\Sigma_{\lambda_{0}}$, we have

$$
u(x) \leq u_{\lambda_{0}}(x) \quad \text { and } \quad v(x) \leq v_{\lambda_{0}}(x), \quad \text { but } u(x) \not \equiv u_{\lambda_{0}}(x) \text { or } v(x) \not \equiv v_{\lambda_{0}}(x) .
$$


We show that the plane can be moved further up. More precisely, there exist an $\epsilon>0$ depending on $n, \alpha$, and the solution $(u(x), v(x))$ such that

$$
u(x) \leq u_{\lambda}(x) \quad \text { and } \quad v(x) \leq v_{\lambda}(x) \quad \text { on } \Sigma_{\lambda} \text { for all } \lambda \text { in }\left[\lambda_{0}, \lambda_{0}+\epsilon\right) .
$$

In the case

$$
v(x) \not \equiv v_{\lambda_{0}}(x) \quad \text { on } \Sigma_{\lambda_{0}}
$$

by Lemma 2.2, we have in fact $u(x)<u_{\lambda_{0}}(x)$ in the interior of $\Sigma_{\lambda_{0}}$. Let

$$
\bar{\Sigma}_{\lambda_{0}}^{u}=\left\{x \in \Sigma_{\lambda_{0}} \mid u(x) \geq u_{\lambda_{0}}(x)\right\} \quad \text { and } \quad \bar{\Sigma}_{\lambda_{0}}^{v}=\left\{x \in \Sigma_{\lambda_{0}} \mid v(x) \geq v_{\lambda_{0}}(x)\right\} .
$$

Then obviously $\bar{\Sigma}_{\lambda_{0}}^{u}$ has measure zero, and $\lim _{\lambda \rightarrow \lambda_{0}} \Sigma_{\lambda}^{u} \subset \bar{\Sigma}_{\lambda_{0}}^{u}$. The same is true for that of $v$. From (3.7) and (3.8), we deduce

$$
\left\|u_{\lambda}-u\right\|_{L^{r}\left(\Sigma_{\lambda}^{u}\right)} \leq C\|v\|_{L^{q_{1}}\left(\Sigma_{\lambda}^{\nu}\right.}^{q-1}\|u\|_{L^{p_{1}}\left(\Sigma_{\lambda}^{u}\right)}^{p-1}\left\|u_{\lambda}-u\right\|_{L^{r}\left(\Sigma_{\lambda}^{u}\right)} .
$$

Again the conditions that $u \in L^{p_{1}}\left(R_{+}^{n}\right)$ and $v \in L^{q_{1}}\left(R_{+}^{n}\right)$ ensure that one can choose $\epsilon$ sufficiently small, so that for all $\lambda$ in $\left[\lambda_{0}, \lambda_{0}+\epsilon\right)$,

$$
C\|v\|_{L^{q_{1}\left(\Sigma_{\lambda}^{\nu}\right)}}^{q-1}\|u\|_{L^{p_{1}\left(\Sigma_{\lambda}^{u}\right)}}^{p-1} \leq \frac{1}{2} .
$$

Now by (3.11), we have $\left\|u_{\lambda}-u\right\|_{L^{p_{1}}\left(\Sigma_{\lambda}^{u}\right)}=0$, therefore $\Sigma_{\lambda}^{u}$ must have measure zero. Similarly, $\Sigma_{\lambda}^{v}$ must also have measure zero. This verifies (3.10), therefore both $u(x)$ and $v(x)$ are symmetric about the plane $T_{\lambda_{0}}$.

Next, we will show that the plane cannot stop at $x_{n}=\lambda_{0}$ for some $\lambda_{0}<+\infty$, that is, we will prove that $\lambda_{0}=+\infty$.

Suppose that $\lambda_{0}<+\infty$, Theorem 1.3 shows that the plane $x_{n}=2 \lambda_{0}$ entails the symmetric points of the boundary $\partial R_{+}^{n}$ with respect to the plane $T_{\lambda_{0}}$, and we derive $u(x)=0$ and $v(x)=0$ when $x$ is on the plane $x_{n}=2 \lambda_{0}$. This contradicts the pair of positive solutions $(u(x), v(x))$ of $(1.4)$, thus $\lambda_{0}=+\infty$. Also the monotonicity easily follows from the argument. This completes the proof of Theorem 1.3.

Proof of Theorem 1.4 We know that both $u(x)$ and $v(x)$ of positive solutions of (1.4) are strictly monotonically increasing in the positive direction of $x_{n}$-axis, but $u \in L^{p_{1}}\left(R_{+}^{n}\right)$ and $v \in L^{q_{1}}\left(R_{+}^{n}\right)$, so we come to the conclusion that the pair of positive solutions $(u(x), v(x))$ of (1.4) does not exist.

This completes the proof of the Theorem 1.4.

\section{Some examples of the pair $\left(p_{1}, q_{1}\right)$ concerning Liouville-type theorems}

One would naturally ask the existence of such pairs of $\left(p_{1}, q_{1}\right)$ that satisfy all these conditions (1.7), (1.8), and (1.9) in Theorem 1.4, here we present some examples to answer the question.

Example 1 In the special case where $p=q$ and $u(x)=v(x)$, system (1.4) becomes the following single integral equation:

$$
u(x)=\int_{R_{+}^{n}} G(x, y, \alpha) u^{p}(y) d y, \quad x \in R_{+}^{n} .
$$


The first and the second authors in [4] obtained the following Liouville-type theorem.

Theorem 4.1 ([4]) Suppose $p>\frac{n}{n-\alpha}$. If the solution $u$ of (4.1) satisfies $u \in L^{\frac{n(p-1)}{\alpha}}\left(R_{+}^{n}\right)$ and is nonnegative, then $u \equiv 0$.

Compared with Theorem 1.4, Theorem 4.1 is the special case where $p_{1}=q_{1}=\frac{n(p-1)}{\alpha}$.

Example 2 The first and the second authors in [3] also considered system (1.4) under the critical case (1.5) and obtained Theorem 1.2. We could find that Theorem 1.2 is coincident with the special case $p_{1}=p+1, q_{1}=q+1$.

Example 3 In [5], the authors discussed the more general integral system

$$
\left\{\begin{array}{l}
u(x)=\int_{R_{+}^{n}} G(x, y, \alpha)|y|^{-s} v^{q}(y) d y, \\
v(x)=\int_{R_{+}^{n}} G(x, y, \alpha)|y|^{-t} u^{p}(y) d y, \quad \forall x \in R_{+}^{n} .
\end{array}\right.
$$

They considered the case when $p$ and $q$ are both subcritical, that is,

$$
p<2 *(t)-1=: \frac{2(n-t)}{n-\alpha}-1 \text { and } q<2 *(s)-1=: \frac{2(n-s)}{n-\alpha}-1,
$$

and they showed the Liouville-type theorem as follows.

Theorem 4.2 ([5]) Suppose that $u(x), v(x) \in C^{1}\left(R^{n}\right)$ are positive solutions of (4.2) with (4.3). If $\int_{R_{+}^{n}} \frac{u^{p+1}}{|x|^{t}} d x<\infty, \int_{R_{+}^{n}} \frac{v^{q+1}}{|x|^{s}} d x<\infty$ and $\alpha-s>1, \alpha-t>1$, then $u \equiv 0$ and $v \equiv 0$.

Now consider the special case $s=t=0$ in (4.2), and system (4.2) reduces to the simple system (1.4). For convenience we rewrite Theorem 4.2 as follows.

Theorem 4.3 Suppose that $u(x), v(x) \in C^{1}\left(R^{n}\right)$ are positive solutions of (1.4) with $p$ and $q$ are both subcritical, that is, $p, q<\frac{n+\alpha}{n-\alpha}$. Assume that $u \in L^{p+1}\left(R_{+}^{n}\right)$ and $v \in L^{q+1}\left(R_{+}^{n}\right)$ are nonnegative, then $u=v \equiv 0$.

Theorem 4.3 above is just Theorem 1.4 when $p_{1}=p+1, q_{1}=q+1$.

Remark 3 Both Theorem 1.2 and Theorem 4.3 are special cases when $p_{1}=p+1, q_{1}=q+1$ in Theorem 1.4, the former concerns the critical case and the latter the subcritical case for system (1.4). 


\section{Acknowledgements}

The authors would like to express their gratitude to the referees for valuable comments and suggestions. Besides, this work is partially supported by the National Natural Science Foundation of China (No. U1304101; No. 11171091) and NSF of Henan Provincial Education Committee (No. 132300410141).

Received: 14 January 2014 Accepted: 25 April 2014 Published: 12 May 2014

\section{References}

1. Chen, W, Li, C: An integral system and the Lane-Emden conjecture. Discrete Contin. Dyn. Syst. 4, 1167-1184 (2009)

2. Chen, W, Li, C: Super polyharmonic property of solutions for PDE systems and its applications. Commun. Pure Appl. Anal. 12(6), 2497-2514 (2013)

3. Cao, L, Dai, Z: A Liouville-type theorem for an integral system on a half-space $R_{+}^{n}$. J. Inequal. Appl. 2013, 37 (2013). doi:10.1186/1029-242X-2013-37

4. Cao, L, Dai, Z: A Liouville-type theorem for an integral equation on a half-space $R_{+}^{n}$. J. Math. Anal. Appl. 389, 1365-1373 (2012)

5. Li, D, Niu, P, Zhuo, R: Symmetry and nonexistence of positive solutions for PDE system with Navier boundary conditions on a half space. Complex Var. Elliptic Equ. (2013). doi:10.1080/17476933.2013.854346

10.1186/1029-242X-2014-173

Cite this article as: Cao et al.: Liouville-type theorem for some nonlinear systems in a half-space. Journal of Inequalities and Applications 2014, 2014:173

\section{Submit your manuscript to a SpringerOpen ${ }^{\circ}$ journal and benefit from:}

- Convenient online submission

- Rigorous peer review

Immediate publication on acceptance

Open access: articles freely available online

- High visibility within the field

- Retaining the copyright to your article 